\title{
Synthesis of tri- and tetramines containing two 2,3-dihydroxypyrrolidine moieties and their inhibitory activity toward $\alpha$-mannosidases
}

\author{
Sandrine Gerber-Lemaire, ${ }^{* a}$ Florence Popowycz, ${ }^{a}$ Eliazar Rodriguez-García, ${ }^{a}$ Catherine \\ Schütz, ${ }^{\mathrm{a}}$ Ana T. Carmona Asenjo, ${ }^{\mathrm{b}}$ Inmaculada Robina, ${ }^{\mathrm{b}}$ and Pierre Vogel $^{\mathrm{a}}$ \\ ${ }^{a}$ Institut de chimie moléculaire et biologique, Ecole Polytechnique Fédérale de Lausanne, BCH, \\ CH-1015 Lausanne, Switzerland and ${ }^{b}$ Departamento de Química Orgánica, Facultad de \\ Química, Universidad de Sevilla, E-41071 Sevilla, Spain \\ E-mail: Sandrine.Gerber@epfl.ch
}

\section{Dedicated to Professor Josef Muchowski on the occasion of his $65^{\text {th }}$ birthday}

(received 20 Feb 03; accepted 08 Apr 03; published on the web 16 Apr 03)

\begin{abstract}
Through the reductive amination of $N$-[(tert-butoxy)carbonyl]-2,5-dideoxy-2,5-imino-3,4-Oisopropylidene-L-ribose with tetramethylenediamine, hexamethylenediamine, 2,7diaminofluorene, 4,4'-diaminodiphenylmethane and 1,4-(diaminomethyl)benzene, five tetramines containing two $(2 R, 3 R, 4 S)$-2-aminomethylpyrrolidine-3,4-diol moieties have been prepared and assayed for their inhibitory activities toward 24 glycosidases. Tetramines containing the tetramethylene or benzene-1,4-dimethylene linkers are more potent $\alpha$ mannosidase inhibitors than simple $(2 R, 3 R, 4 S)$-2-aminomethylpyrrolidine-3,4-diols. Triamines such as $(2 S, 3 R, 4 S)$-bis(3,4-dihydroxy-pyrrolidin-2-ethyl)amine were also prepared and shown to be better $\alpha$-mannosidase inhibitors than $(2 S, 3 R, 4 S)-2$-(2-aminoethyl)pyrrolidin-3,4-diol.
\end{abstract}

Keywords: $\alpha$-Mannosidase inhibitors, polyamines containing hydroxylated pyrrolidines, reductive amination

\section{Introduction}

Cell sociology involves a language based on molecular recognition between cell-surface carbohydrates and proteins. ${ }^{1}$ The biosynthesis of the surface oligosaccharides uses glycosyltransferases and glycosidases as catalysts. Inhibitors of these enzymes ${ }^{2}$ are important molecular tools for glycobiology, and can be used to modulate cellular functions. They are also potential drugs in new therapeutic strategies. ${ }^{3}$ Among the most potent glycosidase inhibitors are polyhydroxypiperidines (1,5-dideoxy-1,5-iminoalditols) that are mimics of the glycosyl cation 
intermediates liberated during enzyme-catalyzed hydrolytic processes. ${ }^{4,5}$ Derivatives of 3,4dihydroxypyrrolidines (1,4-dideoxy-1,4-iminoalditols) also emerge as an important class of glycosidase $^{4 a, 5,6}$ and glycosyltransferase ${ }^{7}$ inhibitors. Simple meso-3,4-dihydroxypyrrolidine $\mathbf{1}$ is a non-selective, weak inhibitor of several glycosidases (Figure 1). ${ }^{8}$ We have found that derivatives 2b with (2R)-aminomethyl side chains can be highly selective and competitive inhibitors of $\alpha$ mannosidases, especially for $\mathrm{Ar}=$ phenyl, thiophenyl. $^{8}$

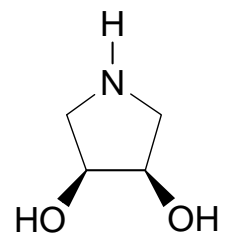

1

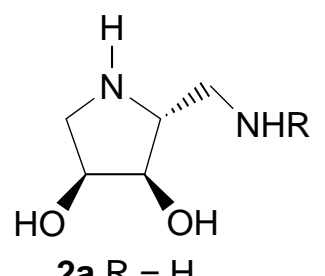

2a $\mathrm{R}=\mathrm{H}$

2b $\mathrm{R}=\mathrm{CH}_{2} \mathrm{Ar}$

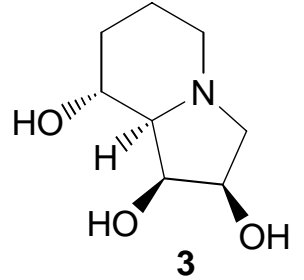

3

Figure 1. Inhibitors of glycosidases and glycosyltransferases.

Clinical trials have shown that swainsonine 3, a natural $\alpha$-mannosidase inhibitor that contains a 4-amino-4-deoxy-mannofuranoside moiety, ${ }^{9,10}$ reduces solid tumors and hematological malignancies. ${ }^{11}$ Analogues of $\mathbf{3}$ have also shown interesting properties. ${ }^{12}$ Mannosidase inhibitors mediate increased secretion of mutant $\alpha 1$-antitrypsin Z. They are thus leads in the development of drugs for the chemoprophylaxis of liver injury and emphysema in patients with $\alpha 1$-antitrypsin $\mathrm{Z}$ deficiency. ${ }^{13}$ Mannostatin A and B isolated from the soil microorganism Streptoverticillum verticillus $^{14}$ and a synthetic analogue ${ }^{15}$ are probably the most potent inhibitors of $\alpha$ mannosidases reported so far. ${ }^{16}$ Often $\alpha$-mannosidase inhibitors that are monosaccharide mimics $^{4 a, 17}$ also inhibit other types of glycosidases, ${ }^{18}$ in particular $\alpha$-L-fucosidases. ${ }^{4 a, 19}$ To become a drug, a good inhibitor must satisfy a number of conditions apart from its low toxicity and enzyme specificity. ${ }^{20}$ We have envisioned that polyamines containing two $(2 R, 3 R, 4 S)-2$ (aminomethyl)-3,4-dihydroxypyrrolidine fragments could be alternative $\alpha$-mannosidase inhibitors with improved pharmacological properties. We report here the synthesis of five tetramines 4 (Figure 2). We have also prepared triamine 5 that contains two $(2 S, 3 R, 4 S)-2-(1-$ aminoeth-2-yl)-3,4-dihydroxypyrrolidine moieties, as well as its enantiomer ent-5. These new compounds have been assayed for their inhibitory activity toward 24 commercially available glycosidases, and in particular toward $\alpha$-mannosidase from jack bean, an enzyme known to be a useful model for mammalian $\alpha$-mannosidases such as Golgi $\alpha$-mannosidase II. ${ }^{21}$ Whereas triamine ent-5 does not inhibit any of the enzyme tested (except for a poor 38\% inhibition of $\beta$ glucosidase from almond at $1 \mathrm{mM}$ concentration), its enantiomer 5 is a moderate inhibitor of $\alpha$ mannosidase from jack bean $\left(K_{i}=74 \mu \mathrm{M}\right)$ and from almond $\left(K_{i}=92 \mu \mathrm{M}\right)$. Among the five tetramines $\mathbf{4}$, best inhibitory activities toward these enzymes were found with $\mathbf{4 a}$ and $\mathbf{4 e}$. But contrary to inhibitors of type $\mathbf{2} \mathbf{b}$, these polyamines are less enzyme selective. 


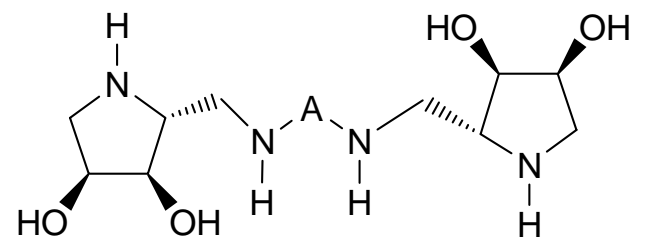

4
a: $A=\left(\mathrm{CH}_{2}\right)_{4}$
b: $A=\left(\mathrm{CH}_{2}\right)_{6}$

c: $A=$<smiles>[AsH2]</smiles>

d:<smiles>[12Cl]c1ccc2c(c1)Cc1cc([TeH])ccc1-2</smiles><smiles>OCc1ccc(C[Te]=C=[Te])cc1</smiles><smiles>O[C@H]1C[C@@H](O)[C@@H](O)[C@H]1NCC[C@@H]1[C@@H](O)CN[C@@H]1CCN[TlH]</smiles>

5<smiles>O[C@H]1CN[C@H](CCNCC[C@H]2NC[C@H](O)[C@@H]2O)[C@@H]1O</smiles>

ent-5

Figure 2. Tri- and tetramines containing two 2,3-dihydroxypyrrolidine moieties.

\section{Results and Discussion}

\section{Synthesis of the polyamines}

Tetramines 4 were all prepared from aldehyde $6^{8}$ by reaction with the corresponding diamine $\mathrm{H}_{2} \mathrm{~N}-\mathrm{A}-\mathrm{NH}_{2}$ (1.8 equivalent) in the presence of $\mathrm{NaBH}(\mathrm{OAc})_{3}{ }^{22}$ for in situ reduction of the resulting diimine intermediate (Scheme 1).

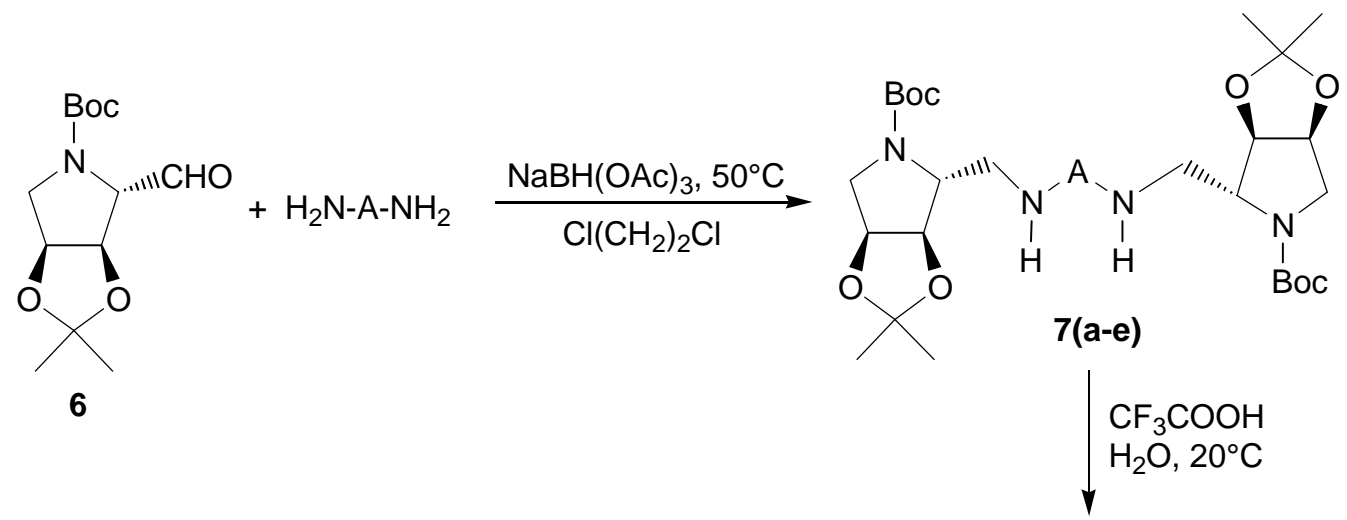

4(a-e), 50 to $90 \%$ yield

Scheme 1. Synthesis of tetramines 4. 
The so-formed semi-protected tetramines were treated with aqueous $\mathrm{CF}_{3} \mathrm{COOH}$, at room temperature, to cleave the Boc and acetonide moieties. Overall yields based on 6 ranged from 50 to $90 \%$.

Triamines $\mathbf{5}$ and ent-5 were derived from aldehydes $\mathbf{8}$ and ent-8, themselves derived from Land D-arabinose, respectively ${ }^{23,24}$ (Scheme 2). Treatment of a 1:1.1 mixture of 8 and benzylamine with $\mathrm{NaBH}(\mathrm{OAc})_{3}$ in 1,2-dichloroethane resulted in the formation of $\mathbf{9}$ and $\mathbf{1 0}$ with $46 \%$ and $18 \%$ yield, respectively. Using a half equivalent of benzylamine, 10 was obtained in $55 \%$ yield. Hydrogenolysis of the benzyl group (10\% Pd / charcoal, THF/MeOH) gave $\mathbf{1 1}$ in $98 \%$ yield. Deprotection under acidic conditions provided 5 in almost quantitative yield. The same reactions were applied to ent-8 providing ent-(9-13). Compound ent-8 was obtained from known $1 \mathbf{1 4}^{24}$ after Boc-protection and reduction with DIBAL-H.

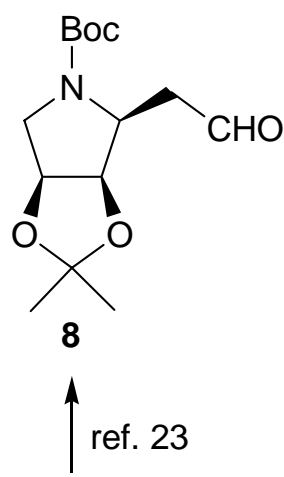

L-arabinose
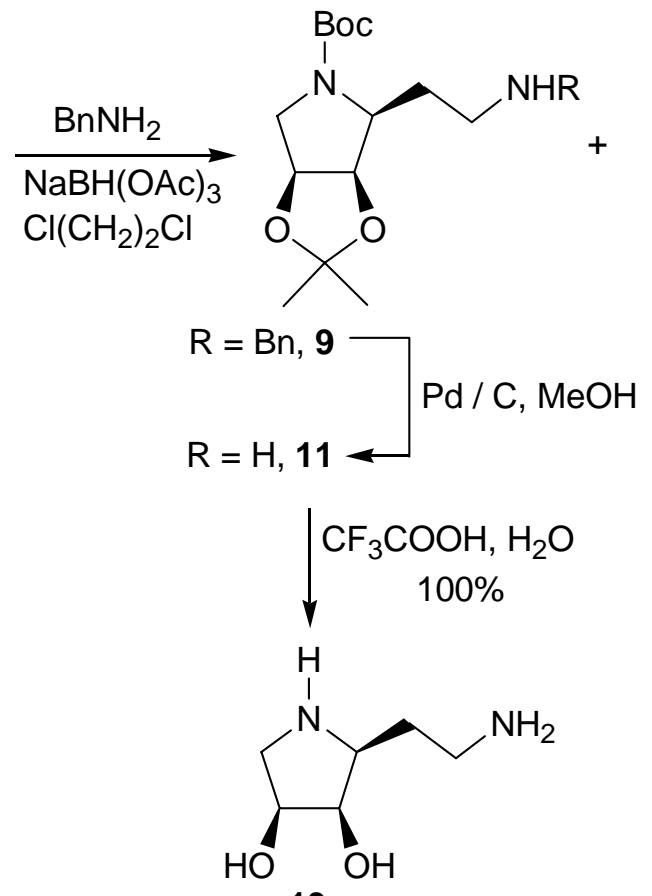
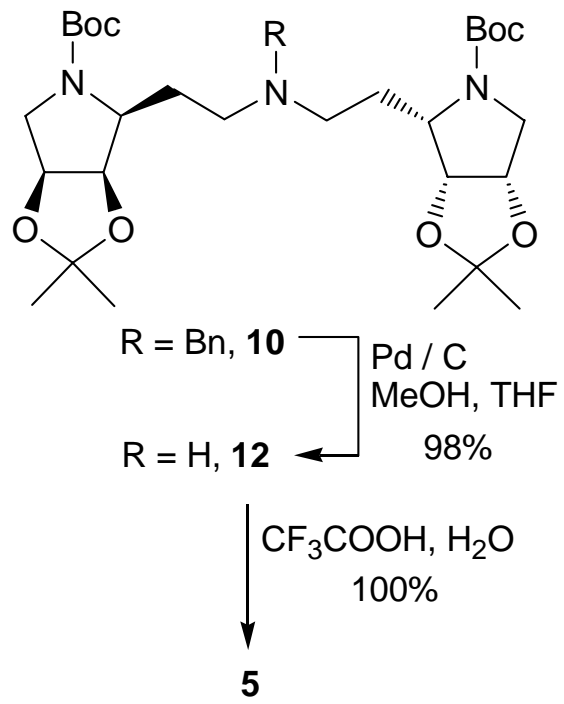

13

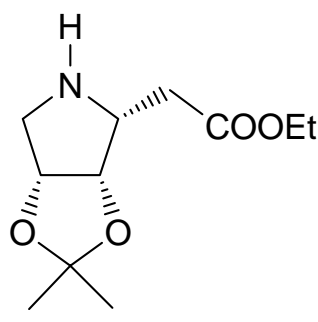

14

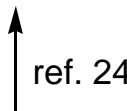

D-arabinose
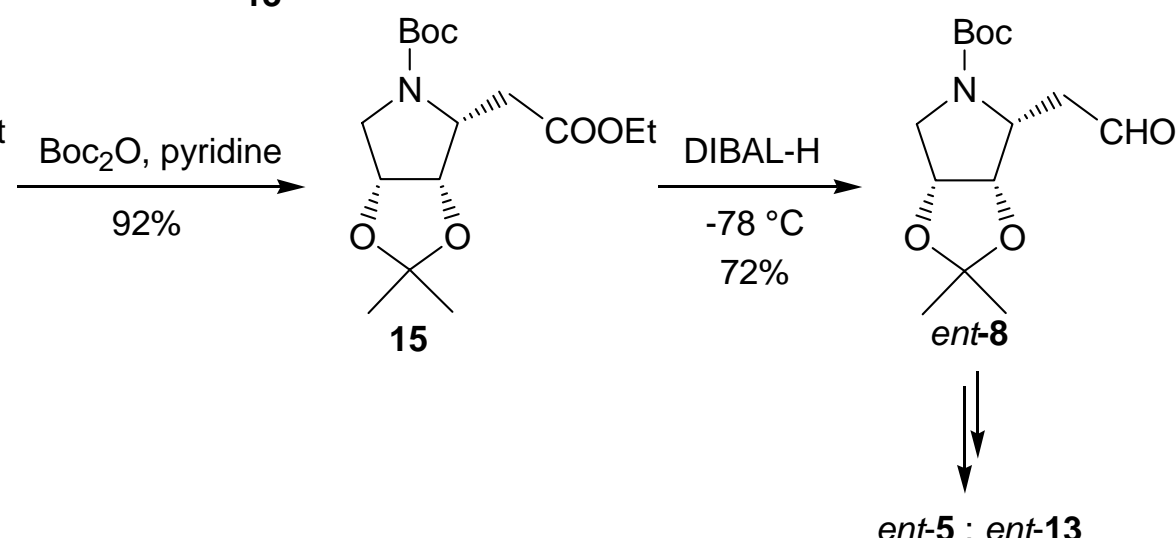

Scheme 2. Preparation of triamines 5 and ent-5. 


\section{Glycosidase inhibitory activities}

Appropriate p-nitrophenyl pyranosides were used as substrates and commercially available glycosidases (see below and Table) were used as catalysts of the buffered hydrolysis under optimal $\mathrm{pH}^{25}$ At $1 \mathrm{mM}$ concentration and under optimal $\mathrm{pH}$ conditions tetramines 4 and triamines 5 and ent-5 did not inhibit the following enzymes: $\alpha$-L-fucosidase from bovine epididymis, $\alpha$-D-galactosidases from coffee bean, Aspergillus niger and E. coli, $\beta$-galactosidase from orizae, $\beta$-D-mannosidase from Helix pomatia, $\beta$ - $N$-acetylgalactosamidase from jack bean, bovine epididymis $\mathrm{A}$ and $\mathrm{B}$. The inhibitory activities toward other glycosidases are reported in Table 1.

We have found that $(2 R, 3 R, 4 S)$-2-aminomethylpyrrolidine-3,4-diol $\mathbf{2 a}$ is a weak inhibitor of $\alpha$-mannosidase from jack bean and from almond. This diamine also moderately inhibits $\beta$ galactosidases, $\alpha$-glucosidases and $\beta$-glucosidases. Derivatives $\mathbf{2 b}$ are much better and more selective $\alpha$-mannosidase inhibitors. ${ }^{8}$ Thus, we expected that compounds $\mathbf{4}$ and $\mathbf{5}$ would also show improved inhibitory activities toward $\alpha$-mannosidases. This is indeed the case for $\mathbf{4 a}$ with the tetramethylene linker, and for $\mathbf{4 e}$ with the $p$-benzenedimethylene spacer. Both are competitive inhibitors. The bad surprise is that these tetramines also inhibit other glycosidases, moderately though, except for $\mathbf{4 a}$ which is a good, non-competitive inhibitor of $\beta$-glucosidase from almond. This result suggests that 4a "sticks" to this enzyme and inhibits it for allosteric reasons, a mechanism different from that making $4 \mathbf{a}$ a competitive inhibitor of $\alpha$-mannosidases. Tetramine 4b with the hexamethylene linker and analogues $\mathbf{4 c}$ and $\mathbf{4 d}$ with diphenylmethane linkers are poor inhibitors in terms of both potency and selectivity. They are even worse than simple diamine 2a. As $(2 S, 3 R, 4 S)$-2-(2-aminoethyl)pyrrolidine-3,4-diol $\mathbf{1 3}$ is a weak inhibitor of $\alpha$-mannosidase, although the side chain is in a $\beta$-configuration rather than $\alpha$, we envisioned that triamine 5 might have improved inhibitory activity. Interestingly, we find 5 to be a more potent $\alpha$-mannosidase inhibitor than 13. Unfortunately, it is not a more selective inhibitor than 13 because it inhibits moderately a few $\alpha$-glucosidases, $\beta$-glucosidases and $\alpha$-N-acetylgalactosamidase from chicken liver (Table 1). As expected, triamine ent-5, which does not share the configuration of any of the hexoses liberated during the hydrolytical process catalyzed by the enzymes used in this study, ignores all these glycosidases.

\section{Conclusions}

The conjugation of two $(2 R, 3 R, 4 S)$-2-(2-aminomethyl)pyrrolidine-3,4-diols by their primary amines to alkane or arene linkers can generate potent $\alpha$-mannosidase inhibitors. This work opens a new road in the search for new glycosidase inhibitors. Analogues of tetramines $\mathbf{4 a}$ and $\mathbf{4 e}$ that will be more enzyme selective remain to be made. 
Table 1. Inhibitory activities of diamines $\mathbf{2 a}, \mathbf{2} \mathbf{b}$, triamines $\mathbf{5}$ and ent-5 and tetramines $4 \mathbf{a}-\mathbf{4 e}$. Percentage of inhibition at $1 \mathrm{mM}$ concentration, $\mathrm{IC}_{50}$ (in parenthesis) and $\mathrm{Ki}$ in $\mu \mathrm{M}$, optimal $\mathrm{pH}$, $35^{\circ} \mathrm{C}^{25,26}$

\begin{tabular}{|c|c|c|c|c|c|c|c|c|c|}
\hline Enzyme / inhibitor & $2 \mathrm{a}$ & $2 b$ & $4 a$ & $4 b$ & $4 \mathrm{c}$ & $4 d$ & $4 \mathrm{e}$ & 5 & ent-5 \\
\hline \multicolumn{10}{|l|}{$\beta$-galactosidase from } \\
\hline E-coli & $92 \%$ & $24 \%$ & $95 \%$ & $43 \%$ & $47 \%$ & ni & $37 \%$ & ni & ni \\
\hline bovine liver & ni & $26 \%$ & $\mathrm{ni}$ & $24 \%$ & $95 \%$ & $82 \%$ & $41 \%$ & ni & ni \\
\hline Aspergillus niger & $24 \%$ & ni & ni & ni & ni & ni & $40 \%$ & $22 \%$ & ni \\
\hline jack bean & $76 \%$ & ni & $45 \%$ & $23 \%$ & ni & ni & $39 \%$ & $31 \%$ & ni \\
\hline \multicolumn{10}{|l|}{$\alpha$-glucosidase from } \\
\hline yeast (maltase) & $24 \%$ & ni & $88 \%$ & $37 \%$ & ni & $\mathrm{ni}$ & $55 \%$ & ni & ni \\
\hline rice (maltase) & $53 \%$ & ni & $\mathrm{ni}$ & $\mathrm{ni}$ & $26 \%$ & ni & ni & ni & ni \\
\hline baker yeast (isomaltase) & $98 \%$ & ni & $\mathrm{ni}$ & $69 \%$ & ni & ni & $86 \%$ & $50 \%$ & ni \\
\hline $\begin{array}{c}\text { Aspergillus niger } \\
\text { (amyloglucosidase) }\end{array}$ & ni & ni & ni & ni & ni & ni & $28 \%$ & $26 \%$ & ni \\
\hline $\begin{array}{c}\text { Rhyzopus mold } \\
\text { (amyloglucosidase) }\end{array}$ & $\mathrm{ni}$ & ni & ni & ni & $26 \%$ & ni & $39 \%$ & ni & ni \\
\hline \multicolumn{10}{|l|}{$\beta$-glucosidase from } \\
\hline almonds & $97 \%$ & $68 \%$ & $97 \%(160)$ & $87 \%(110)$ & $35 \%$ & $52 \%$ & $85 \%(99)$ & $37 \%$ & $38 \%$ \\
\hline $\mathrm{Ki}=$ & & & $8(\mathrm{NC})$ & $110(\mathrm{C})$ & & & $65(\mathrm{C})$ & & \\
\hline caldocellum sacch. & $93 \%$ & ni & $90 \%$ & $76 \%$ & $36 \%$ & $29 \%$ & $67 \%$ & $26 \%$ & $\mathrm{ni}$ \\
\hline \multicolumn{10}{|l|}{$\alpha$-mannosidase from } \\
\hline jack bean & $81 \%$ & $92 \%$ & $76 \%(330)$ & $72 \%$ & ni & $47 \%$ & $95 \%(50)$ & $71 \%(300)$ & ni \\
\hline $\mathrm{Ki}=$ & $53(\mathrm{C})$ & $7.4(\mathrm{C})$ & $21(\mathrm{C})$ & & & & $12(\mathrm{C})$ & $74(\mathrm{C})$ & \\
\hline almonds & $51 \%$ & $69 \%$ & $85 \%(92)$ & $70 \%$ & $39 \%$ & ni & $81 \%(145)$ & $65 \%(280)$ & ni \\
\hline $\mathrm{Ki}=$ & & $7(\mathrm{C})$ & $10(\mathrm{C})$ & & & & $48(\mathrm{C})$ & $92(\mathrm{C})$ & \\
\hline \multicolumn{10}{|l|}{$\beta$-xylosidase from } \\
\hline Aspergillus niger & ni & $\mathrm{ni}$ & $\mathrm{ni}$ & $\mathrm{ni}$ & $\mathrm{ni}$ & $\mathrm{ni}$ & $26 \%$ & ni & ni \\
\hline \multicolumn{10}{|l|}{$\alpha-N$-acetylgalactosamidase } \\
\hline chicken liver & ni & ni & ni & $92 \%(100)$ & ni & $\mathrm{ni}$ & $91 \%(53)$ & ni & ni \\
\hline $\mathrm{Ki}=$ & & & & $43(\mathrm{C})$ & & & $31(\mathrm{C})$ & & \\
\hline
\end{tabular}

$\mathrm{ni}=$ no inhibition, $\mathrm{C}=$ competitive, $\mathrm{NC}=$ non-competitive

\section{Experimental Section}

General Procedures. All commercially available reagents (Fluka, Aldrich) were used without further purification. Solvents were dried by standard methods. Light petroleum ether used refers to the fraction boiling at $40-60{ }^{\circ} \mathrm{C}$. Solutions after reactions and extractions were evaporated in a 
rotatory evaporator under reduced pressure. Liquid/solid flash chromatography (FC): columns of silica gel (Merck No.9385 silica gel 60, 240-400 mesh). TLC for reaction monitoring: Merck silica gel $60 \mathrm{~F}_{254}$ plates; detection by UV light, Pancaldi reagent $\left[\left(\mathrm{NH}_{4}\right)_{6} \mathrm{MoO}_{4}, \mathrm{Ce}\left(\mathrm{SO}_{4}\right)_{2}, \mathrm{H}_{2} \mathrm{SO}_{4}\right.$, $\mathrm{H}_{2} \mathrm{O}$ ] or $\mathrm{KMnO}_{4}$. IR spectra: Perkin-Elmer-1420 spectrometer. Optical rotations were determined at room temperature on a Jasco DIP-370 polarimeter. $[\alpha]_{D}$ values are given in units of $10^{-1} \mathrm{deg} \mathrm{cm}^{2} \mathrm{~g}^{-1}$. ${ }^{1}$ H NMR spectra: Bruker-ARX-400 spectrometer (400 MHz), Bruker AMX-300 spectrometer $(300 \mathrm{MHz}) ; \delta(\mathrm{H})$ in ppm relative to the solvent's residual ${ }^{1} \mathrm{H}$ signal $\left[\mathrm{CHCl}_{3}, \delta(\mathrm{H}) 7.27 ; \mathrm{CH}_{3} \mathrm{OD}\right.$, $\delta(\mathrm{H}) 3.31 ; \mathrm{D}_{2} \mathrm{O}, \delta(\mathrm{H}) 4.79$; DMSO- $\left.d_{6}, \delta(\mathrm{H}) 2.54\right]$ as internal reference; all ${ }^{1} \mathrm{H}$ assigments were confirmed by $2 \mathrm{D}-\mathrm{COSY}-45$ and $2 \mathrm{D}-\mathrm{NOESY}$ spectra. ${ }^{13} \mathrm{C}$ NMR spectra: same instrument as above $(100.6 \mathrm{MHz}$ and $75.4 \mathrm{MHz}) ; \delta(\mathrm{C})$ in ppm relative to the solvent's $\mathrm{C}$-signal $\left[\mathrm{CDCl}_{3}, \delta(\mathrm{C})\right.$ 77.0; $\mathrm{CD}_{3} \mathrm{OD}, \delta(\mathrm{C}) 49.8$; DMSO- $\left.d_{6} \delta(\mathrm{C}) 39.7\right]$ as internal reference; all ${ }^{13} \mathrm{C}$ assigments were confirmed by 2D-HMQC; coupling constants $J$ in Hz. MS: Nermag R 10-10C, chemical ionization $\left(\mathrm{NH}_{3}\right)$ mode $\mathrm{m} / \mathrm{z}(\mathrm{amu})$ [\% relative to base peak (100\%)]. High resolution mass spectrometry: Micromass AutoSpecQ, resolution of 10000 (5\% valley definition). Elemental analyses: Ilse Beetz, D-96301 Kronach, Germany.

Glycosidase inhibitions. A known protocol was applied. ${ }^{25,26}$ We verified that the delay of inhibitor/enzyme incubation did not affect the inhibition measurements. Under standard conditions, optimal inhibitory activities were measured after five minutes of incubation.

Reductive amination. General procedure A. To a solution of $N$-[(t-butoxy)carbonyl $]-2,5-$ dideoxy-2,5-imino-3,4-O-isopropylidene-L-ribose $(200 \mathrm{mg}, 0.737 \mathrm{mmol})$ in anhydrous 1,2-dichloroethane $(7 \mathrm{~mL})$ were added the diamine $(0.6 \mathrm{eq}, 0.442 \mathrm{mmol})$ and $\mathrm{NaBH}(\mathrm{OAc})_{3}(1.8 \mathrm{eq}$, $281 \mathrm{mg}, 1.327 \mathrm{mmol})$. The solution was stirred at $50^{\circ} \mathrm{C}$ for $12 \mathrm{~h}$ and then poured into a sat. aq solution of $\mathrm{NaHCO}_{3}(20 \mathrm{~mL})$. The mixture was extracted with EtOAc $(3 \times 20 \mathrm{~mL})$. The combined organic extracts were dried $\left(\mathrm{MgSO}_{4}\right)$ and concentrated under reduced pressure. The residue was directly used in the deprotection step.

Reductive amination. General procedure B. To a solution of $N$-[(t-butoxy)carbonyl] $-2,3,6-$ trideoxy-3,6-imino-4,5-O-isopropylidene-L- (or D-) arabino-hexose (1 mmol) in anhydrous 1,2dihloroethane $(3 \mathrm{~mL})$ were added benzylamine $(118 \mathrm{mg}, 1.1 \mathrm{mmol})$ and $\mathrm{NaBH}(\mathrm{OAc})_{3}(276 \mathrm{mg}$, $1.3 \mathrm{mmol})$. The solution was stirred at r.t. for $3 \mathrm{~h}$ and then poured into a sat. aq solution of $\mathrm{NaHCO}_{3}(20 \mathrm{~mL})$. The mixture was extracted with EtOAc $(3 \times 20 \mathrm{~mL})$ and the combined organic extracts were dried $\left(\mathrm{MgSO}_{4}\right)$. After solvent evaporation under reduced pressure the residue was purified by flash chromatography on silica gel $\left(\mathrm{CH}_{2} \mathrm{Cl}_{2} / \mathrm{MeOH}\right.$ 60:1 to 5:1).

Deprotection. General procedure C. A solution of bis (pyrrolidine) derivatives in $\mathrm{CF}_{3} \mathrm{COOH} /$ $\mathrm{H}_{2} \mathrm{O}(4: 1 ; 5-10 \%)$ was stirred at $20^{\circ} \mathrm{C}$ for $2 \mathrm{~h}$. After solvent evaporation in vacuo, the residue was purified by flash chromatography on silica gel $\left(\mathrm{MeCN} / \mathrm{aq} \mathrm{NH} \mathrm{N}_{3}\right)$. 
Deprotection. General procedure D. A solution of the protected pyrrolidine derivative $(0.1 \mathrm{mmol})$ in $\mathrm{CF}_{3} \mathrm{COOH} \mathrm{H} \mathrm{H}_{2} \mathrm{O}(4: 1 ; 3 \mathrm{~mL})$ was stirred at $20^{\circ} \mathrm{C}$ for $2 \mathrm{~h}$. The mixture was passed through a Dowex 50WX8 (100-200 mesh) column and eluted, successively with $\mathrm{MeOH}$ $(30 \mathrm{~mL}), \mathrm{H}_{2} \mathrm{O}(30 \mathrm{~mL})$ and $\mathrm{NH}_{4} \mathrm{OH}(10 \%, 50 \mathrm{~mL})$. The fractions containing the unprotected product were concentrated to yield the corresponding pyrrolidine derivative.

(2R,3R,4S)-2-[[4-[[[(2R,3R,4S)-3,4-Dihydroxypyrrolidin-2-yl]methyl]amino]butyl]aminomethyl]pyrrolidine-3,4-diol (4a). Procedure A was applied to 1,4-diaminobutane (45 $\mu \mathrm{L}$, $0.442 \mathrm{mmol})$ to afford crude $7 \mathbf{a}(180 \mathrm{mg})$. Deprotection according to procedure $\mathrm{C}$ gave 4a (127 mg, 90\%, 2 steps) as a pale orange oil. $\mathrm{R}_{f}=0.15\left(\mathrm{MeCN} / \mathrm{NH}_{4} \mathrm{OH} 1: 1\right) .[\alpha]_{589}^{25}=-106$, $[\alpha]_{577}^{25}=-262,[\alpha]_{546}^{25}=-409,[\alpha]_{435}^{25}=-690,[\alpha]_{405}^{25}=-1114\left(c=0.25, \mathrm{H}_{2} \mathrm{O}\right)$. IR (film): $\widetilde{v} 3500-$ 2900, 1440, 1200, 1140, 840, 800, 710, $695 \mathrm{~cm}^{-1}$. UV (MeCN): $\lambda_{\max }(\varepsilon) 195$ (1360). ${ }^{1} \mathrm{H}$ NMR $\left(\mathrm{D}_{2} \mathrm{O}\right): \delta 4.11\left(\mathrm{~m}, 2 \mathrm{H}, \mathrm{H}-4, \mathrm{H}-4^{\mathrm{IV}}\right), 3.94\left(\mathrm{dd}, 2 \mathrm{H},{ }^{3} \mathrm{~J}=10.7,{ }^{3} \mathrm{~J}=3.9 \mathrm{~Hz}, \mathrm{H}-3, \mathrm{H}-3^{\mathrm{IV}}\right.$ ), 3.75 (ddd, $\left.2 \mathrm{H},{ }^{3} \mathrm{~J}=10.7,{ }^{3} \mathrm{~J}=5.1,{ }^{3} \mathrm{~J}=3.3 \mathrm{~Hz}, \mathrm{H}-2, \mathrm{H}-2^{\mathrm{IV}}\right), 3.23\left(\mathrm{dd}, 2 \mathrm{H},{ }^{2} J=9.3,{ }^{3} J=2.1, \mathrm{H}-5, \mathrm{H}-5^{\mathrm{IV}}\right)$, $3.13\left(\mathrm{dd}, 2 \mathrm{H},{ }^{2} \mathrm{~J}=12.6,{ }^{3} \mathrm{~J}=5.1, \mathrm{H}-1^{\prime}, \mathrm{H}-1^{\prime \prime \prime}\right), 3.09-3.01\left(\mathrm{~m}, 2 \mathrm{H}, \mathrm{H}-5, \mathrm{H}-5^{\mathrm{IV}}\right), 2.81\left(\mathrm{dd}, 2 \mathrm{H},{ }^{2} J=\right.$ 12.6, ${ }^{3} \mathrm{~J}=3.3$, H-1', H-1"'), 2.72-2.64 (m, 4H, H-1", H-4"), 1.71-1.56 (m, 4H, H-2", H-3"). ${ }^{13} \mathrm{C}$ NMR $\left(\mathrm{D}_{2} \mathrm{O}\right): \delta 76.9\left(\mathrm{~d}, \mathrm{C}-3, \mathrm{C}-3^{\mathrm{IV}}\right), 73.5\left(\mathrm{~d}, \mathrm{C}-4, \mathrm{C}-4^{\mathrm{IV}}\right), 60.7\left(\mathrm{~d}, \mathrm{C}-2, \mathrm{C}-2^{\mathrm{IV}}\right), 53.9(\mathrm{t}, \mathrm{C}-5, \mathrm{C}-$ $\left.5^{\mathrm{IV}}\right), 52.6$ (t, C-1, C-1"'), 50.4 (t, C-1", C-4"), 26.3 (t, C-2", C-3"). CI-MS: m/z 319 (100, M + $\mathrm{H}^{+}$), 293 (74), 204 (33), 133 (35), 102 (36), 84 (55). Anal. calcd for $\mathrm{C}_{14} \mathrm{H}_{30} \mathrm{~N}_{4} \mathrm{O}_{4}$ (318.42): C, 52.81; H, 9.50. Found: C, 52.79; H, 9.32 .

(2R,3R,4S)-2-[[6-[[[(2R,3R,4S)-3,4-Dihydroxypyrrolidin-2-yl]methyl]aminohexyl]aminomethyl]pyrrolidine-3,4-diol (4b). Procedure A was applied to 1,6-diaminohexane (51 mg, $0.442 \mathrm{mmol})$ to afford crude $\mathbf{7 b}(155 \mathrm{mg})$. Deprotection according to procedure $\mathrm{C}$ gave $\mathbf{4 b}$ (86 mg, 56\% yield, 2 steps) as a colorless oil. $\mathrm{R}_{f}=0.1\left(\mathrm{MeCN}, \mathrm{NH}_{4} \mathrm{OH} 1 / 1\right) .[\alpha]_{589}^{25}=-54(\mathrm{c}=$ 0.5, $\mathrm{H}_{2} \mathrm{O}$ ). IR (film): $\widetilde{v}$ 3500-2900, 1450, 1195, 1150, 840, 800, 705, $700 \mathrm{~cm}^{-1}$. UV (MeCN): $\lambda_{\max }(\varepsilon) 197$ (1450). ${ }^{1} \mathrm{H}$ NMR $\left(\mathrm{D}_{2} \mathrm{O}\right): \delta 4.18\left(\mathrm{~m}, 2 \mathrm{H}, \mathrm{H}-4, \mathrm{H}-4^{\mathrm{IV}}\right), 3.95\left(\mathrm{dd}, 2 \mathrm{H},{ }^{3} J=5.4,{ }^{3} J=2.7\right.$ $\left.\mathrm{Hz}, \mathrm{H}-3, \mathrm{H}-3^{\mathrm{IV}}\right), 3.75$ (m, 2H, H-2, H- $\left.2^{\mathrm{IV}}\right), 3.29$ (m, 2H, H-5, H-5 $\left.{ }^{\mathrm{IV}}\right), 3.152 \mathrm{H},\left(2 \mathrm{H}, \mathrm{dd},{ }^{2} \mathrm{~J}=13.2\right.$, $\left.{ }^{3} J=4.8, \mathrm{H}-1^{\prime}, \mathrm{H}-1^{\prime \prime \prime}\right), 3.09$ (m, 2H, H-5, H-5 $\left.{ }^{\mathrm{IV}}\right), 2.94$ (dd, $\left.2 \mathrm{H},{ }^{2} J=13.2,{ }^{3} J=3.1, \mathrm{H}-1^{\prime}, \mathrm{H}-1^{\prime \prime \prime}\right)$, 2.73 (m, 4H, H-1", H-6"), 1.65-1.54 (m, 4H, H-2", H-5"), 1.38 (m, 4H, H-3", H-4"). ${ }^{13} \mathrm{C}$ NMR $\left(\mathrm{D}_{2} \mathrm{O}\right): \delta 77.0\left(\mathrm{~d}, \mathrm{C}-3, \mathrm{C}-3^{\mathrm{IV}}\right), 71.7\left(\mathrm{~d}, \mathrm{C}-4, \mathrm{C}-4^{\mathrm{IV}}\right), 60.7\left(\mathrm{~d}, \mathrm{C}-2, \mathrm{C}-2^{\mathrm{IV}}\right), 54.2\left(\mathrm{t}, \mathrm{C}-5, \mathrm{C}-5^{\mathrm{IV}}\right), 52.6$ (t, C-1, C-1"'), 50.9 (t, C-1", C-6"), 29.7 (t, C-2", C-5"), 26.3 (t, C-3", C-4"). CI-MS: m/z 347 (28, $\mathrm{M}+\mathrm{H}^{+}$), 274 (9), 232 (12), 117 (100), 98 (85), 86 (63). Anal. calcd for $\mathrm{C}_{16} \mathrm{H}_{34} \mathrm{~N}_{4} \mathrm{O}_{4}$ (346.47): C, 55.47; H, 9.89; N, 16.17. Found: C, 55.18; H, 9.70; N, 16.01.

\section{(2R,3R,4S)-2-[4-[4-[I[(2R,3R,4S)-3,4-Dihydroxypyrrolidin-2-yl]methyl]amino]benzyl]phenyl-} aminomethyl]pyrrolidine-3,4-diol (4c). Procedure A was applied to 4,4'-diaminodiphenylmethane $(88 \mathrm{mg}, 0.442 \mathrm{mmol})$ to afford crude $7 \mathrm{c}(150 \mathrm{mg})$. Deprotection according to procedure $\mathrm{C}$ gave 4c (113 mg, 60\% yield, 2 steps) as a pale yellow oil. $\mathrm{R}_{f}=0.10\left(\mathrm{MeCN} / \mathrm{NH}_{4} \mathrm{OH} 4: 1\right)$. $[\alpha]_{589}^{25}=+27,[\alpha]_{577}^{25}=+34,[\alpha]_{546}^{25}=+41(\mathrm{c}=0.9, \mathrm{MeOH})$. IR (film): $\widetilde{v} 3400-3200,2950,1675$, 1515, 1450, 1205, 1140, 1025, $725 \mathrm{~cm}^{-1}$. UV (MeCN): $\lambda_{\max }(\varepsilon) 260$ (7250), 207 (13980). ${ }^{1} \mathrm{H}$ NMR (MeOD): $\delta 6.94,6.68\left(2 \mathrm{~d}, 8 \mathrm{H},{ }^{3} J=8.5 \mathrm{~Hz}, \mathrm{H}-2^{\prime}, \mathrm{H}-6 ", \mathrm{H}-3^{\mathrm{VI}}, \mathrm{H}-5^{\mathrm{VI}}\right), 4.29$ (m, 2H, H-4, $\left.\mathrm{H}-4^{\mathrm{VI}}\right), 4.08\left(\mathrm{dd}, 2 \mathrm{H},{ }^{3} \mathrm{~J}=8.6,{ }^{3} \mathrm{~J}=4.0 \mathrm{~Hz}, \mathrm{H}-3, \mathrm{H}-3^{\mathrm{VI}}\right), 3.80$ (bs, $\left.2 \mathrm{H}, 2 \mathrm{H}-1{ }^{\prime \prime \prime}\right), 3.76$ (ddd, $2 \mathrm{H},{ }^{3} J=$ 
9.1, $\left.{ }^{3} J=8.6,{ }^{3} J=3.7 \mathrm{~Hz}, \mathrm{H}-2, \mathrm{H}-2^{\mathrm{VI}}\right), 3.60\left(\mathrm{dd}, 2 \mathrm{H},{ }^{2} J=14.4,{ }^{3} \mathrm{~J}=3.7 \mathrm{~Hz}, \mathrm{H}-1^{\prime}, \mathrm{H}-1^{\mathrm{V}}\right), 3.48$ (dd, $\left.2 \mathrm{H},{ }^{2} \mathrm{~J}=14.4,{ }^{3} \mathrm{~J}=4.0 \mathrm{~Hz}, \mathrm{H}-5, \mathrm{H}-5^{\mathrm{VI}}\right), 3.45\left(\mathrm{dd}, 2 \mathrm{H},{ }^{2} \mathrm{~J}=14.4,{ }^{3} \mathrm{~J}=3.7 \mathrm{~Hz}, \mathrm{H}-1^{\prime}, \mathrm{H}-1^{\mathrm{V}}\right), 3.27$ $\left(\mathrm{dd}, 2 \mathrm{H},{ }^{2} J=14.4,{ }^{3} J=1.9 \mathrm{~Hz}, \mathrm{H}-5, \mathrm{H}-5^{\mathrm{VI}}\right.$ ). ${ }^{13} \mathrm{C}$ NMR (MeOD): $\delta 133.3$ (s, C-1", C-4 ${ }^{\mathrm{IV}}$ ), 133.2 (d, C-2", C-6", C-3 $\left.{ }^{\mathrm{IV}}, \mathrm{C}-5^{\mathrm{IV}}\right), 122.3$ (s, C-4", C-1 $\left.{ }^{\mathrm{IV}}\right), 116.9$ (d, C-3", C-5", C-2 $\left.2^{\mathrm{IV}}, \mathrm{C}-6^{\mathrm{IV}}\right), 77.3$ (d, $\left.\mathrm{C}-3, \mathrm{C}-3^{\mathrm{VI}}\right), 73.5\left(\mathrm{~d}, \mathrm{C}-4, \mathrm{C}-4^{\mathrm{VI}}\right), 64.0\left(\mathrm{~d}, \mathrm{C}-2, \mathrm{C}-2^{\mathrm{VI}}\right), 53.3\left(\mathrm{t}, \mathrm{C}-5, \mathrm{C}-5^{\mathrm{VI}}\right), 47.1$ (t, C-1', C-1 $\left.{ }^{\mathrm{V}}\right)$, 43.8 (t, C-1"'). Anal. calcd for $\mathrm{C}_{23} \mathrm{H}_{30} \mathrm{~N}_{4} \mathrm{O}_{4}$ (426.51): C, 64.77; H, 7.09; N, 13.14. Found: C, $64.34 ; \mathrm{H}, 7.28 ; \mathrm{N}, 12.99$.

(2R,3R,4S)-2-[[7-[[[(2R,3R,4S)-3,4-Dihydroxypyrrolidin-2-yl]methyl]amino]-9H-fluoren-2yl]aminomethyl]pyrrolidine-3,4-diol (4d). Procedure A was applied with 2,7-diaminofluorene $(87 \mathrm{mg}, 0.442 \mathrm{mmol})$ to afford crude $7 \mathbf{d}(153 \mathrm{mg})$. Deprotection according to procedure $\mathrm{C}$ gave 4d (94 mg, 50\% yield, 2 steps) as a pale yellow oil. $\mathrm{R}_{f}=0.09\left(\mathrm{MeCN} / \mathrm{NH}_{4} \mathrm{OH} 2 / 1\right)$. $[\alpha]_{589}^{25}=-62$, $[\alpha]_{577}^{25}=-74,[\alpha]_{546}^{25}=-103,[\alpha]_{435}^{25}=-107,[\alpha]_{405}^{25}=-130(c=1, \mathrm{MeOH})$. IR (film): $\widetilde{v} 3400-$ 3200, 2960, 1675, 1520, 1455, 1210, 1135, 125, 880, $765 \mathrm{~cm}^{-1}$. UV (MeCN): $\lambda_{\max }(\varepsilon) 308$ (5820), 215 (5680), 203 (6200). ${ }^{1} \mathrm{H}$ NMR (MeOD): $\delta 7.43$ (d, 2H, ${ }^{3} J=8.1 \mathrm{~Hz}, \mathrm{H}-3 "$, H-6"), 6.92 (bs, 2H, H-1", H-8"), 6.71 (d, 2H, $\left.{ }^{3} J=8.1 \mathrm{~Hz}, \mathrm{H}-4 ", \mathrm{H}-5 "\right), 4.32$ (m, 2H, H-4, H-4 ${ }^{\mathrm{IV}}$ ), 4.13 (dd, $\left.2 \mathrm{H},{ }^{3} \mathrm{~J}=8.5,{ }^{3} \mathrm{~J}=4.0 \mathrm{~Hz}, \mathrm{H}-3, \mathrm{H}-3^{\mathrm{IV}}\right), 3.82\left(\mathrm{ddd}, 2 \mathrm{H},{ }^{3} J=8.5,{ }^{3} J=8.4,{ }^{3} \mathrm{~J}=3.8 \mathrm{~Hz}, \mathrm{H}-2, \mathrm{H}-2^{\mathrm{IV}}\right)$, 3.73 (bs, 2H, H-9"), 3.67 (dm, 2H, $\left.{ }^{2} J=13.3 \mathrm{~Hz}, \mathrm{H}-5, \mathrm{H}-5^{\mathrm{IV}}\right), 3.52$ (m, 2H, H-5, H-5 ${ }^{\mathrm{IV}}$ ), 3.51 (dd, $\left.2 \mathrm{H},{ }^{2} \mathrm{~J}=12.6,{ }^{3} \mathrm{~J}=4.0 \mathrm{~Hz}, \mathrm{H}-1^{\prime}, \mathrm{H}-1^{\prime \prime \prime}\right), 3.30\left(\mathrm{dd}, 2 \mathrm{H},{ }^{2} \mathrm{~J}=12.6,{ }^{3} \mathrm{~J}=1.8 \mathrm{~Hz}, \mathrm{H}-1\right.$ ', H-1"'). ${ }^{13} \mathrm{C}$ NMR (MeOD): $\delta$ 148.2, 146.2 (2s, C-4a", C-4b", C-8a", C-9a"), 121.0 (s, C-2", C-7"), 120.5 (d, C-3", C-6"), 114.2 (d, C-4", C-5"), 111.9 (d, C-1", C-8"), 75.7 (d, C-3, C-3 ${ }^{\mathrm{IV}}$ ), 71.9 (d, C-4, C$\left.4^{\mathrm{IV}}\right), 62.5\left(\mathrm{~d}, \mathrm{C}-2, \mathrm{C}-2^{\mathrm{IV}}\right), 51.7$ (t, C-1', C-1"'), 45.7 (d, C-5, C-5 $\left.{ }^{\mathrm{IV}}\right), 42.1$ (t, C-9"). CI-MS: $\mathrm{m} / \mathrm{z}$ $427\left(21, \mathrm{M}+\mathrm{H}^{+}\right.$), 370 (14), 311 (50), 197 (28), 98 (100), 80 (57). Anal. calcd for $\mathrm{C}_{23} \mathrm{H}_{30} \mathrm{~N}_{4} \mathrm{O}_{4}$ (426.51): C, 64.77; H, 7.09. Found: C, 64.88; H, 7.23.

(2R,3R,4S)-2-[[4-[[[(2R,3R,4S)-3,4-Dihydroxypyrrolidin-2-yl]methyl]aminomethyl]benzyl]aminomethyl]pyrrolidine-3,4-diol (4e). Procedure A was applied with 1,4-(diaminomethyl)benzene $(60 \mathrm{mg}, 0.442 \mathrm{mmmol})$ to afford crude 7e $(157 \mathrm{mg})$. Deprotection according to procedure $\mathrm{C}$ gave $4 \mathbf{e}(89 \mathrm{mg}, 55 \%$ yield, 2 steps $)$ as a colorless oil. $\mathrm{R}_{f}=0.14\left(\mathrm{MeCN} / \mathrm{NH}_{4} \mathrm{OH}\right.$ $1: 1) .[\alpha]_{589}^{25}=+57,[\alpha]_{577}^{25}=+77,[\alpha]_{546}^{25}=+83,[\alpha]_{435}^{25}=+93,[\alpha]_{405}^{25}=+110\left(c=0.65, \mathrm{H}_{2} \mathrm{O}\right)$. IR (film): $\widetilde{v} 3500-3000,1675,1425,1200,1130,835,800,740,700 \mathrm{~cm}^{-1} . \mathrm{UV}(\mathrm{MeCN}): \lambda_{\max }(\varepsilon)$ 197 (5600). ${ }^{1} \mathrm{H}$ NMR ( $\left.\mathrm{D}_{2} \mathrm{O}\right): \delta 7.50$ (bs, 4H, Harom), 4.26 (m, 2H, H-4, H-4 ${ }^{\mathrm{VI}}$ ), 3.94 (s, 4H, 2H-1", $\left.2 \mathrm{H}-1^{\mathrm{IV}}\right), 3.87\left(\mathrm{dd}, 2 \mathrm{H},{ }^{3} \mathrm{~J}=7.6,5.0 \mathrm{~Hz}, \mathrm{H}-3, \mathrm{H}-3^{\mathrm{VI}}\right), 3.30-3.25\left(\mathrm{~m}, 4 \mathrm{H}, \mathrm{H}-2, \mathrm{H}-2^{\mathrm{VI}}, \mathrm{H}-5, \mathrm{H}-5^{\mathrm{VI}}\right)$, 2.99-2.94 (m, 4H, H-1', H-1 ${ }^{\mathrm{V}}, \mathrm{H}-5, \mathrm{H}-5^{\mathrm{VI}}$ ), 2.79 (dd, $2 \mathrm{H},{ }^{2} \mathrm{~J}=12.5,{ }^{3} \mathrm{~J}=8.6 \mathrm{~Hz}, \mathrm{H}-1$ ', H-1V). ${ }^{13} \mathrm{C}$ NMR (D $2 \mathrm{O}): \delta 137.4$ (s, C-1"', C-4"'), 129.2 (d, C-2"', C-3"', C-5"', C-6"'), 75.2 (d, C-3, C-3 ${ }^{\mathrm{VI}}$ ), $70.9\left(\mathrm{~d}, \mathrm{C}-4, \mathrm{C}-4^{\mathrm{VI}}\right), 59.9$ (t, C-1", C-1 $\left.{ }^{\mathrm{IV}}\right), 52.1$ (t, C-5, C-5 $\left.{ }^{\mathrm{VI}}\right), 50.6\left(\mathrm{~d}, \mathrm{C}-2, \mathrm{C}-2^{\mathrm{VI}}\right), 50.0$ (t, C-1', C-1 ${ }^{\mathrm{V}}$ ). CI-MS : m/z $368\left(24, \mathrm{M}^{+}\right), 252$ (7), 133 (100), 117 (59). Anal. calcd for $\mathrm{C}_{18} \mathrm{H}_{32} \mathrm{~N}_{4} \mathrm{O}_{4}$ (368.48): C 58.67; H 8.75; N 15.21. Found: C 58.42, H 8.60, N 15.12.

$\mathrm{N}$-(tert-Butoxycarbonyl)-(2S,3R,4S)-2-[2-(benzylamino)ethyl]-3,4-O-isopropylidenepyrrolidine-3,4-diol (9) and $N, N$-bis[ $N$-(tert-butoxycarbonyl)-[(2S,3R,4S)-3,4-O-isopropylidenoxypyrrolidinyl]ethyl]benzylamine (10). Procedure B was applied to carbaldehyde $\mathbf{8}^{23}$ (298 $\mathrm{mg}$, $1.05 \mathrm{mmol}$ ) affording 9 (178.8 $\mathrm{mg}, 46 \%)$ as oil and 10 (123.4 mg, 18\%) as white solid. 
9. $[\alpha]_{589}^{25}=+46\left(\mathrm{c}=0.94, \mathrm{CH}_{2} \mathrm{Cl}_{2}\right)$. IR $(\mathrm{KBr}): \widetilde{v} 3335,1705,1470,1405,1085,735,695 \mathrm{~cm}^{-1} .{ }^{1} \mathrm{H}$ NMR (DMSO- $\left.d_{6}, 90^{\circ} \mathrm{C}\right): \delta 7.36-7.27\left(\mathrm{~m}, 5 \mathrm{H}, \mathrm{H}_{\text {arom }}\right), 4.71-4.64(\mathrm{~m}, 2 \mathrm{H}, \mathrm{H}-3, \mathrm{H}-4), 3.83(\mathrm{~m}, 1 \mathrm{H}$, $\mathrm{H}-2), 3.75\left(\mathrm{~d}, 1 \mathrm{H},{ }^{2} J=13.6, \mathrm{CH}_{2} \mathrm{Ph}\right), 3.70\left(\mathrm{~d}, 1 \mathrm{H},{ }^{2} J=13.6, \mathrm{CH}_{2} \mathrm{Ph}\right), 3.67\left(\mathrm{dd}, 1 \mathrm{H},{ }^{3} J=7.0,{ }^{2} J=\right.$ 12.2, H-5), 3.13 (dd, $1 \mathrm{H},{ }^{3} J=3.3, \mathrm{H}-5$ ), 3.00 (bs, $1 \mathrm{H}, \mathrm{NH}$ ), 2.65 (ddd, $1 \mathrm{H},{ }^{2} J=11.6, \mathrm{H}-2^{\prime}$ ), 2.59 (ddd, $1 \mathrm{H}, \mathrm{H}-2^{\prime}$ ), 1.93 (dq, $1 \mathrm{H},{ }^{3} J=6.5,{ }^{2} J=13.3, \mathrm{H}-1$ '), 1.81 (dq, $1 \mathrm{H},{ }^{3} J=6.1, \mathrm{H}-1$ ') $) 1.39$ (s, $\left.9 \mathrm{H}, \mathrm{C}\left(\mathrm{CH}_{3}\right)_{3}\right), 1.42$ and $1.26\left(2 \mathrm{~s}, 6 \mathrm{H}, \mathrm{C}\left(\mathrm{CH}_{3}\right)_{2}\right) \cdot{ }^{13} \mathrm{C}$ NMR (DMSO-d $\left.\mathrm{d}_{6} 90^{\circ} \mathrm{C}\right): \delta 153.4(\mathrm{~s}, \mathrm{CO})$, 139.7 (s, Carom), 127.5, 127.4, 125.9 (3d, $\left.\mathrm{C}_{\text {arom }}\right), 111.2$ (s, $\left.\mathrm{C}\left(\mathrm{CH}_{3}\right)_{2}\right), 79.3,76.7$ (2d, C-3, C-4), $78.4\left(\mathrm{~s}, \mathrm{CMe}_{3}\right), 57.3$ (d, C-2), 52.4 (t, $\left.\mathrm{CH}_{2} \mathrm{Ph}\right), 50.0$ (t, C-5), 45.3 (t, C-2'), 28.8 (t, C-1'), 27.6 (q, $\left.\mathrm{C}\left(\mathrm{CH}_{3}\right)_{3}\right), 26.0,24.6\left(2 \mathrm{q}, \mathrm{C}\left(\mathrm{CH}_{3}\right)_{2}\right)$. CI-MS: $\mathrm{m} / \mathrm{z} 377\left(100,[\mathrm{M}+\mathrm{H}]^{+}\right)$. CI-HRMS: $\mathrm{m} / \mathrm{z} 377.2439$ (calcd for $\mathrm{C}_{21} \mathrm{H}_{32} \mathrm{~N}_{2} \mathrm{O}_{4}+\mathrm{H}$ : 223.1446).

10. $[\alpha]_{589}^{25}=+81\left(\mathrm{c}=0.98, \mathrm{CH}_{2} \mathrm{Cl}_{2}\right)$. IR $(\mathrm{KBr}): \widetilde{v} 1700,1400,1165,1100,870,735 \mathrm{~cm}^{-1} .{ }^{1} \mathrm{H}$ NMR (CDCl $\left.{ }_{3}\right): \delta 7.36-7.16\left(\mathrm{~m}, 5 \mathrm{H}, \mathrm{H}_{\text {arom }}\right), 4.49(\mathrm{~m}, 2 \mathrm{H}, \mathrm{H}-4), 4.53\left(\mathrm{dd}, 2 \mathrm{H},{ }^{3} \mathrm{~J}=6.1,{ }^{3} \mathrm{~J}=6.1\right.$, $\mathrm{H}-3$ ), 3.83 (ddd, $\left.2 \mathrm{H},{ }^{3} J=9.3,{ }^{3} J=6.1,{ }^{3} J=5.4, \mathrm{H}-2\right), 3.80\left(\mathrm{~d}, 1 \mathrm{H},{ }^{2} J=12.9, \mathrm{CH}_{2} \mathrm{Ph}\right), 3.77\left(\mathrm{dd},{ }^{2} J\right.$ $\left.=12.1,{ }^{3} J=7.1, \mathrm{H}-5\right), 3.60\left(\mathrm{~d}, 1 \mathrm{H},{ }^{2} J=12.9, \mathrm{CH}_{2} \mathrm{Ph}\right), 3.24\left(\mathrm{dd}, 2 \mathrm{H},{ }^{2} J=12.1,{ }^{3} \mathrm{~J}=4.7, \mathrm{H}-5\right)$, $2.63\left(\mathrm{dt},{ }^{2} J=12.8,{ }^{3} \mathrm{~J}=7.8, \mathrm{H}-2^{\prime}\right), 2.50\left(\mathrm{ddd}, 2 \mathrm{H},{ }^{2} \mathrm{~J}=12.8,{ }^{3} \mathrm{~J}=8.4,{ }^{3} \mathrm{~J}=4.4, \mathrm{H}-2^{\prime}\right), 1.42(\mathrm{~s}, 18 \mathrm{H}$, $\left.{ }^{t} \mathrm{Bu}\right), 1.47,1.27\left(2 \mathrm{~s}, 6 \mathrm{H}, \mathrm{C}\left(\mathrm{CH}_{3}\right)_{2}\right) .{ }^{13} \mathrm{C} \mathrm{NMR}\left(\mathrm{CDCl}_{3}\right): \delta 154.2$ (s, $\left.\mathrm{CO}\right), 140.2$ (s, Carom), 129.0, 127.8, 126.3 (3d, Carom), 112.2 (s, $\left.\mathrm{C}\left(\mathrm{CH}_{3}\right)_{2}\right), 79.3$ (d, C-3), 77.3 (s, $\left.\mathrm{CMe}_{3}\right), 77.3$ (d, C-4), 58.2 (d, C-2), 58.0 (t, $\left.\mathrm{CH}_{2} \mathrm{Ph}\right), 50.7,50.6$ (2t, C-5, C-2'), 28.3 (q, $\left.\mathrm{C}\left(\mathrm{CH}_{3}\right)_{3}\right), 26.5$ (t, C-1'), 26.7, 25.0 $\left(2 \mathrm{q}, \mathrm{C}\left(\mathrm{CH}_{3}\right)_{2}\right)$. CI-MS: $\mathrm{m} / \mathrm{z} 646\left(100, \mathrm{M}+\mathrm{H}^{+}\right)$. CI-HRMS: $\mathrm{m} / \mathrm{z} 646.4069$ (calcd for $\mathrm{C}_{35} \mathrm{H}_{55} \mathrm{~N}_{3} \mathrm{O}_{8}+\mathrm{H}:$ 646.4067).

\section{$\mathrm{N}$-(tert-Butoxycarbonyl)-(2S,3R,4S)-2-aminoethyl-3,4-O-isopropylidenepyrrolidine-3,4-diol}

(11). A solution of $9(131.6 \mathrm{mg}, 0.35 \mathrm{mmol})$ in abs. EtOH $(7 \mathrm{~mL})$ was hydrogenated with catalyst $\mathrm{Pd} / \mathrm{C}(10 \%, 55 \mathrm{mg})$ at $1 \mathrm{~atm}$ for $2 \mathrm{~h}$. The mixture was filtered through Celite, and the filtrate was evaporated to give $11(101 \mathrm{mg}, 100 \%)$ as a syrup. $[\alpha]_{589}^{25}=+48\left(\mathrm{c}=0.6, \mathrm{CH}_{2} \mathrm{Cl}_{2}\right)$. IR (KBr): $\widetilde{v} 1695,1400,1090,800,735 \mathrm{~cm}^{-1} .{ }^{1} \mathrm{H}$ NMR (DMSO- $d_{6}, 90{ }^{\circ} \mathrm{C}$ ): $\delta 4.77-4.70$ (m, 2H, H3, H-4), 3.84 (m, 1H, H-2), 3.68 (dd, $\left.1 \mathrm{H},{ }^{2} J=12.0,{ }^{3} J=7.2, \mathrm{H}-5\right), 3.14$ (dd, $\left.1 \mathrm{H},{ }^{3} J=3.4, \mathrm{H}-5\right)$, 2.66-2.62 (m, 3H, H-2', H-2', NH), 1.87-1.71 (m, 3H, H-1', H-1', NH), 1.43 and 1.29 (2s, 6H $\left.\mathrm{C}\left(\mathrm{CH}_{3}\right)_{2}\right), 1.41\left(\mathrm{~s}, 9 \mathrm{H}, \mathrm{C}\left(\mathrm{CH}_{3}\right)_{3}\right) .{ }^{13} \mathrm{C}$ NMR (DMSO-d $\left.6,90{ }^{\circ} \mathrm{C}\right): \delta 153.4(\mathrm{~s}, \mathrm{CO}), 111.2(\mathrm{~s}$, $\left.\mathrm{C}\left(\mathrm{CH}_{3}\right)_{2}\right), 79.2,76.8$ (2d, C-4, C-3), 78.3 (s, $\left.\mathrm{C}\left(\mathrm{CH}_{3}\right)_{3}\right), 56.9$ (d, C-2), 49.9 (t, C-5), 38.2 (t, C-2'), 32.4 (t, C-1'), 27.6 (q, $\left.\mathrm{C}\left(\mathrm{CH}_{3}\right)_{3}\right), 25.9$ and $24.6\left(2 \mathrm{q}, \mathrm{C}\left(\mathrm{CH}_{3}\right)_{2}\right)$. CI-MS: $\left.\mathrm{m} / \mathrm{z} 287(85 \text {, [M+H] }]^{+}\right)$. CIHRMS: $m / z 287.1971$ (calcd for $\mathrm{C}_{21} \mathrm{H}_{33} \mathrm{~N}_{2} \mathrm{O}_{4}+\mathrm{H}$ : 287.1980).

$N, N$-Bis-[ $N$-(tert-butoxycarbonyl)-[(2S,3R,4S)-3,4-O-isopropylidenoxy-pyrrolidinyl]ethyl]amine (12). A solution of $\mathbf{1 0}(115 \mathrm{mg}, 0.18 \mathrm{mmol})$ in THF/MeOH $(1: 1,4 \mathrm{~mL})$ was hydrogenated with $\mathrm{Pd} / \mathrm{C}(10 \%, 28 \mathrm{mg})$ at $1 \mathrm{~atm}$ for $2.5 \mathrm{~h}$. The mixture was filtered through a pad of Celite and evaporated in vacuo to afford $\mathbf{1 1}$ as a white solid (97 mg, 98\% yield). $[\alpha]_{589}^{25}=+57$ (c $=0.77$, $\mathrm{CH}_{2} \mathrm{Cl}_{2}$ ). IR (KBr): $\widetilde{v} 3335,1705,1470,1405,1085,865,735 \mathrm{~cm}^{-1} .{ }^{1} \mathrm{H}$ NMR (DMSO-d $d_{6}$ ): $\delta$ 4.77-4.70 (m, 4H, H-3, H-4), 3.84 (m, 2H, H-2), 3.68 (dd, $\left.2 \mathrm{H},{ }^{2} J=12.0,{ }^{3} \mathrm{~J}=7.2, \mathrm{H}-5\right), 3.14$ (dd, $\left.2 \mathrm{H},{ }^{2} J=12.0,{ }^{3} \mathrm{~J}=3.4, \mathrm{H}-5\right), 2.66-2.62$ (m, 4H, H-2'), 1.87-1.71 (m, 5H, H-1', NH), 1.43, 1.29

$\left(2 \mathrm{~s}, 6 \mathrm{H}, \mathrm{C}\left(\mathrm{CH}_{3}\right)_{2}\right), 1.41\left(\mathrm{~s}, 18 \mathrm{H}, \mathrm{CMe}_{3}\right) .{ }^{13} \mathrm{C}$ NMR (DMSO-d $\left.\mathrm{d}_{6}\right): \delta 153.4(\mathrm{~s}, \mathrm{CO}), 111.2(\mathrm{~s}$, $\left.\mathrm{C}\left(\mathrm{CH}_{3}\right)_{2}\right), 79.2$ (d, C-3), 78.3 (s, $\mathrm{CMe}_{3}$ ), 76.8 (d, C-4), 56.9 (d, C-2), 49.9 (t, C-5), 38.2 (t, C-2'), 
32.4 (t, C-1'), 27.6 (q, $\left.\mathrm{C}\left(\mathrm{CH}_{3}\right)_{3}\right), 25.9,24.6\left(2 \mathrm{q}, \mathrm{C}\left(\mathrm{CH}_{3}\right)_{2}\right)$. CI-MS: m/z $556\left(100, \mathrm{M}+\mathrm{H}^{+}\right)$. CIHRMS: $m / z 556.3593$ (calcd for $\mathrm{C}_{28} \mathrm{H}_{49} \mathrm{~N}_{3} \mathrm{O}_{8}+\mathrm{H}$ : 556.3598).

(2S, 3R, 4S)-2-Aminoethylpyrrolidine-3,4-diol (13). Deprotection of 11 (94.3 mg, $0.33 \mathrm{mmol})$ according to procedure D gave $13(47 \mathrm{mg}, 98 \%)$ as viscous oil. $[\alpha]_{589}^{25}=+16(\mathrm{c}=1.1, \mathrm{MeOH}) .{ }^{1} \mathrm{H}$ NMR (MeOD): $\delta 4.20$ (m, 1H, H-4), 3.92 (t, 1H, $\left.{ }^{3} J=4.3, \mathrm{H}-3\right), 3.00-2.92$ (m, 2H, H-2, H-5), 2.84-2.77 (m, 3H, H-5b, H-2', H-2'), 1.84 (dq, $1 \mathrm{H},{ }^{2} J=14.0,{ }^{3} J=7.1, \mathrm{H}-1$ '), 1.71 (dq, $1 \mathrm{H},{ }^{3} J=$ 7.0, H-1'). ${ }^{13} \mathrm{C}$ NMR (MeOD): $\delta$ 73.9, 73.4 (2d, C-4, C-3), 60.6 (d, C-2), 51.5 (d, C-5), 39.8 (d, C-2'), 32.2 (d, C-1'). CI-MS: m/z 147 (100, [M+H] $]^{+}$). CI-HRMS: m/z 147.1134 (calcd for $\mathrm{C}_{6} \mathrm{H}_{14} \mathrm{~N}_{2} \mathrm{O}_{2}+\mathrm{H}:$ 147.1135).

$N, N$-Bis-[[(2S, 3R, 4S)-3,4-dihydroxy-pyrrolidinyl]ethyl]amine (5). Deprotection of 12 (100 $\mathrm{mg}, 0.18 \mathrm{mmol}$ ) according to procedure D gave triamine $5(49.5 \mathrm{mg}, 100 \%)$ as viscous oil. $[\alpha]_{589}^{25}=+8(\mathrm{c}=0.5, \mathrm{MeOH})$. IR (KBr): $\widetilde{v} 3295,1690,1460,1410,1095,805 \mathrm{~cm}^{-1} .{ }^{1} \mathrm{H} \mathrm{NMR}$ (MeOD): $\delta 4.20$ (m, 2H, H-4), 3.92 (dd, 2H, ${ }^{3} J=4.3,{ }^{3} J=4.2, \mathrm{H}-3$ ), 3.00-2.92 (m, 4H, H-2, H5), 2.84-2.77 (m, 6H, H-5, H-2'), $1.84\left(\mathrm{dq}, 2 \mathrm{H},{ }^{2} J=14.0,{ }^{3} J=7.1, \mathrm{H}-1\right.$ '), 1.71 (dq, 2H, ${ }^{2} J=14.0$, ${ }^{3} J=7.0, \mathrm{H}-1$ '). ${ }^{13} \mathrm{C}$ NMR (MeOD): $\delta 73.9$ (d, C-3), 73.4 (d, C-4), 60.6 (d, C-2), 51.5 (t, C-5), 39.8 (t, C-2'), 32.2 (t, C-1'). CI-MS: m/z $276\left(80, \mathrm{M}+\mathrm{H}^{+}\right.$). CI-HRMS: m/z 276.1922 (calcd for $\left.\mathrm{C}_{28} \mathrm{H}_{49} \mathrm{~N}_{3} \mathrm{O}_{8}+\mathrm{H}: 276.1923\right)$.

Ethyl $N$-(tert-butoxycarbonyl)-2,3,6-trideoxy-3,6-imino-4,5-O-isopropylidene-D-arabino-2hexanoate (15). To a solution of ethyl 2,3,6-trideoxy-3,6-imino-4,5-O-isopropylidene-Darabino-2-hexanoate $(\mathbf{1 4})^{24}(2.87 \mathrm{~g}, 12.5 \mathrm{mmol})$ in dry pyridine $(35 \mathrm{~mL})$ was added a solution of $(\mathrm{Boc})_{2} \mathrm{O}(3.06 \mathrm{~g}, 13.8 \mathrm{mmol})$ in pyridine $(20 \mathrm{~mL})$. The reaction was left at r.t. for $2 \mathrm{~h}$ and then evaporated. The crude product was dissolved in AcOEt $(100 \mathrm{~mL})$ and washed twice with brine. The organic layer was dried $\left(\mathrm{Na}_{2} \mathrm{SO}_{4}\right)$, filtered and concentrated. Column chromatography of the residue (ether/petroleum ether, 1:5 to 1:2), gave $15(3.78 \mathrm{~g}, 92 \%)$ as an oil. $[\alpha]_{589}^{25}=-68(\mathrm{c}=1.2$, $\mathrm{CH}_{2} \mathrm{Cl}_{2}$ ). IR (KBr): $\widetilde{v}$ 2980, 2940, 1720, 1700, 1380, $1090 \mathrm{~cm}^{-1} .{ }^{1} \mathrm{H}$ NMR (DMSO-d, $90{ }^{\circ} \mathrm{C}$ ): $\delta$ $4.76(\mathrm{~m}, 1 \mathrm{H}, \mathrm{H}-4), 4.73$ (m, 1H, H-5), 4.13 (m, 1H, H-3), 4.08 (q, 2H, $\left.{ }^{2} J=7.1, \mathrm{CH}_{2} \mathrm{CH}_{3}\right), 3.60$ (dd, $\left.1 \mathrm{H},{ }^{3} \mathrm{~J}=6.5,{ }^{2} \mathrm{~J}=12.7, \mathrm{H}-6\right), 3.26\left(\mathrm{dd}, 1 \mathrm{H},{ }^{3} \mathrm{~J}=2.4, \mathrm{H}-6\right.$ '), $2.85\left(\mathrm{dd}, 1 \mathrm{H},{ }^{3} \mathrm{~J}=4.7,{ }^{2} \mathrm{~J}=16.0\right.$, $\mathrm{H}-2), 2.50\left(\mathrm{dd}, 1 \mathrm{H},{ }^{2} \mathrm{~J}=9.6, \mathrm{H}-2\right.$ ') $, 1.40\left(\mathrm{~s}, 9 \mathrm{H}, \mathrm{C}\left(\mathrm{CH}_{3}\right)_{3}\right), 1.41,1.27\left(2 \mathrm{~s}, 6 \mathrm{H}, \mathrm{C}\left(\mathrm{CH}_{3}\right)_{2}\right), 1.19$ (t, $3 \mathrm{H}, \mathrm{CH}_{2} \mathrm{CH}_{3}$ ). ${ }^{13} \mathrm{C} \mathrm{NMR}\left(\mathrm{DMSO}_{-} \mathrm{d}_{6} 90^{\circ} \mathrm{C}\right): \delta 170.0(\mathrm{~s}, \mathrm{CO}), 153.3$ (s, CO of Boc), $111.1(\mathrm{~s}$, $\left.\mathrm{C}\left(\mathrm{CH}_{3}\right)_{2}\right), 78.9$ (d, C-4), $78.7\left(\mathrm{~s}, \mathrm{CMe}_{3}\right), 76.9$ (d, C-5), 59.0 (t, $\left.\mathrm{CH}_{2} \mathrm{CH}_{3}\right), 56.0$ (d, C-3), 50.0 (t, C-6), 33.8 (t, C-2), 27.6 (q, C( $\left.\left(\mathrm{CH}_{3}\right)_{3}\right), 25.4,24.5\left(2 \mathrm{q}, \mathrm{C}\left(\mathrm{CH}_{3}\right)_{2}\right), 13.4$ (q, $\left.\mathrm{CH}_{2} \mathrm{CH}_{3}\right)$. CI-MS: $\mathrm{m} / \mathrm{z}$ $330\left(60,[\mathrm{M}+\mathrm{H}]^{+}\right)$. Anal. calcd for $\mathrm{C}_{16} \mathrm{H}_{27} \mathrm{NO}_{6}$ (329.39): C, 58.34; H, 8.26; N, 4.25. Found: C, 58.49; H, 8.16; N, 4.32.

\section{$\mathrm{N}$-(tert-Butoxycarbonyl)-2,3,6-trideoxy-3,6-imino-4,5-O-isopropyliden-D-arabinohexose}

(ent-8). To a solution of ethyl $N$-(tert-butoxycarbonyl)-2,3,6-trideoxy-3,6-imino-4,5-O-isopropyliden-D-arabino-2-hexanoate (15) (0.76 g, $2.32 \mathrm{mmol})$ in dry dichloromethane (10 $\mathrm{mL})$, was added dropwise a solution of DIBAL-H in dichloromethane $(1 \mathrm{M}, 4.6 \mathrm{~mL}, 4.6 \mathrm{mmol})$ at $78^{\circ} \mathrm{C}$ under Ar. After $2 \mathrm{~h}$ at $-78^{\circ} \mathrm{C} \mathrm{MeOH}(4 \mathrm{~mL})$ was slowly added, and the reaction mixture was left to warm up to r.t. Then the mixture was cooled to $0{ }^{\circ} \mathrm{C}, \mathrm{HCl}(1 \mathrm{M}, 10 \mathrm{~mL})$ was added, and the mixture was extracted with dichloromethane $(4 \times 50 \mathrm{~mL})$. The organic layer was washed 
with saturated aqueous $\mathrm{NaHCO}_{3}$, dried $\left(\mathrm{Na}_{2} \mathrm{SO}_{4}\right)$, filtered and evaporated. Column chromatography of the residue (ether/petroleum ether $1: 4$ to $1: 2)$ gave ent-8 $(0.48 \mathrm{~g}, 72 \%)$ as viscous oil. $[\alpha]_{589}^{25}=80\left(\mathrm{c}=0.68, \mathrm{CH}_{2} \mathrm{Cl}_{2}\right)$. IR $(\mathrm{KBr}): \widetilde{v} 2935,1725,1400,1090 \mathrm{~cm}^{-1} .1 \mathrm{H} \mathrm{NMR}$ (DMSO-d $\left.6,90{ }^{\circ} \mathrm{C}\right): \delta 9.69\left(\mathrm{t}, 1 \mathrm{H},{ }^{3} \mathrm{~J}=1.7, \mathrm{CHO}\right), 4.79-4.71(\mathrm{~m}, 2 \mathrm{H}, \mathrm{H}-4, \mathrm{H}-5), 4.21\left(\mathrm{c}, 1 \mathrm{H},{ }^{3} \mathrm{~J}=\right.$ 6.6, H-3), $3.60\left(\mathrm{dd}, 1 \mathrm{H},{ }^{3} \mathrm{~J}=6.4,{ }^{2} \mathrm{~J}=12.3, \mathrm{H}-6\right), 3.29\left(\mathrm{dd}, 1 \mathrm{H},{ }^{3} \mathrm{~J}=2.3, \mathrm{H}-6\right.$ ') 2.74 (dd, 2H, H-2, H-2'), 1.40 (s, 9H, C(CH $\left.)_{3}\right), 1.41,1.27\left(2 \mathrm{~s}, 6 \mathrm{H}, \mathrm{C}\left(\mathrm{CH}_{3}\right)_{2}\right) .{ }^{13} \mathrm{C}$ NMR (DMSO-d $\left.6,90{ }^{\circ} \mathrm{C}\right): \delta 200.0$ (s, CHO), $153.4(\mathrm{~s}, \mathrm{CO}), 111.1\left(\mathrm{~s}, \mathrm{C}\left(\mathrm{CH}_{3}\right)_{2}\right), 79.0$, 76.8 (2d, C-4, C-5), 78.9 (s, $\left.\mathrm{CMe}_{3}\right), 55.2$ (d, C-3), 50.3 (t, C-6), 42.9 (t, C-2), $27.6\left(\mathrm{q}, \mathrm{C}\left(\mathrm{CH}_{3}\right)_{3}\right), 25.6$ and $24.5\left(2 \mathrm{q}, \mathrm{C}\left(\mathrm{CH}_{3}\right)_{2}\right) . \mathrm{FAB}-\mathrm{MS}: \mathrm{m} / \mathrm{z}$ $286\left(20,[\mathrm{M}+\mathrm{H}]^{+}\right)$. Anal. calcd for $\mathrm{C}_{14} \mathrm{H}_{23} \mathrm{NO}_{5}$ (285.34): C, 58.93; H, 8.12; N, 4.91. Found: C, 58.69; H, 8.39; N, 5.16.

$N$-(tert-Butoxycarbonyl)-(2R,3S,4R)-2-[2-(benzylamino)ethyl]-3,4-O-isopropylidenepyrrolidine-3,4-diol (ent-9) and $N, N$-bis[ $N$-(tert-butoxycarbonyl)-[(2R,3S,4R)-3,4-O-isopropylidenoxypyrrolidinyl]ethyl]benzylamine (ent-10). Procedure B was applied to carbaldehyde ent-8 (327 mg, $1.15 \mathrm{mmol})$ to afford ent-9 (155.3 mg, 36\%) as an oil and ent-10 (95.5 mg, 13\%) as a white solid.

ent-9. $[\alpha]_{589}^{25}=-47\left(\mathrm{c}=0.7, \mathrm{CH}_{2} \mathrm{Cl}_{2}\right)$. CI-HRMS: $\mathrm{m} / \mathrm{z} 377.2439$ (calcd for $\mathrm{C}_{21} \mathrm{H}_{32} \mathrm{~N}_{2} \mathrm{O}_{4}+\mathrm{H}$ : 377.2446).

ent-10. $[\alpha]_{589}^{25}=-88\left(\mathrm{c}=0.54, \mathrm{CH}_{2} \mathrm{Cl}_{2}\right)$. CI-MS: $m / z 668\left(40, \mathrm{M}+\mathrm{NH}_{4}{ }^{+}\right), 646\left(60, \mathrm{M}+\mathrm{H}^{+}\right)$. CIHRMS: $m / z 646.4057$ (calcd for $\mathrm{C}_{35} \mathrm{H}_{55} \mathrm{~N}_{3} \mathrm{O}_{8}+\mathrm{H}$ : 646.4067). NMR and IR spectra were identical to those of its enantiomer $\mathbf{1 0 .}$

$N$-(tert-Butoxycarbonyl)-(2R,3S,4R)-2-aminoethyl-3,4-O-isopropylidene-pyrrolidine-3,4-diol (ent-11). A solution of ent-9 $(146.3 \mathrm{mg}, 0.39 \mathrm{mmol})$ in abs. EtOH $(8 \mathrm{~mL})$ was hydrogenated with catalyst $\mathrm{Pd} / \mathrm{C}(10 \%)(62 \mathrm{mg})$ at $1 \mathrm{~atm}$ for $2 \mathrm{~h}$. The mixture was filtered through Celite and the filtrate was evaporated to give ent-11 $(111 \mathrm{mg}, 100 \%)$ as syrup. $[\alpha]_{589}^{25}=-55\left(\mathrm{c}=0.8, \mathrm{CH}_{2} \mathrm{Cl}_{2}\right)$. CIMS: m/z 287 [50\%, (M+H) ${ }^{+*}$. CI-NSHR: $m / z 287.1963$ (cald for $\mathrm{C}_{14} \mathrm{H}_{26} \mathrm{~N}_{2} \mathrm{O}_{4}+\mathrm{H}$ : 287.1971). This product showed NMR and IR spectra identical to those of its enantiomer 11.

$N, N$-Bis[ $N$-(tert-butoxycarbonyl)-[(2R,3S,4R)-3,4-O-isopropylidenoxy-pyrrolidinyl]ethyl] amine (ent-12). A solution of ent-10 (90 mg, $0.14 \mathrm{mmol})$ in THF-MeOH (1.5 mL / $1.5 \mathrm{~mL})$ was hydrogenated for $1.5 \mathrm{~h}$ under $1 \mathrm{~atm}$ with $\mathrm{Pd} / \mathrm{C}(10 \%$ on charcoal, $22 \mathrm{mg})$. The mixture was filtered through a pad of Celite and concentrated in vacuo to afford ent-11 (78 mg, 100\%) as white solid. $[\alpha]_{589}^{25}=-62\left(\mathrm{c}=0.45, \mathrm{CH}_{2} \mathrm{Cl}_{2}\right)$. CI-MS: $\mathrm{m} / \mathrm{z} 556\left(100, \mathrm{M}+\mathrm{H}^{+}\right)$. CI-HRMS: $\mathrm{m} / \mathrm{z}$ 556.3589 (calcd for $\mathrm{C}_{28} \mathrm{H}_{49} \mathrm{~N}_{3} \mathrm{O}_{8}+\mathrm{H}$ : 556.3598). NMR and IR spectra were identical to those of its enantiomer 12.

(2R,3S,4R)-2-Aminoethylpyrrolidine-3,4-diol (ent-13). Deprotection of ent-11 (102 mg, $0.36 \mathrm{mmol})$ according to procedure $\mathrm{D}$ gave ent-13 $(52 \mathrm{mg}, 91 \%)$ as thick oil. $[\alpha]_{589}^{25}=-12(\mathrm{c}=$ 0.1, MeOH). CI-HRMS m/z 147.1136 (calcd for $\mathrm{C}_{6} \mathrm{H}_{14} \mathrm{~N}_{2} \mathrm{O}_{2}+\mathrm{H}$ : 147.1134). This product showed NMR spectra identical to those of its enantiomer 13.

$\boldsymbol{N}, \boldsymbol{N}$-bis-[[(2R,3S,4R)-3,4-Dihydroxypyrrolidinyl]ethyl]amine (ent-5). Deprotection of ent-12 $(77 \mathrm{mg}, 0.14 \mathrm{mmol})$ according to procedure D gave ent-5 $(37 \mathrm{mg}, 97 \%)$ as oil. $[\alpha]_{589}^{25}=-10(\mathrm{c}=$ 
0.78, MeOH). CI-MS: m/z $276\left(80, \mathrm{M}+\mathrm{H}^{+}\right.$). CI-HRMS m/z 276.1919 (calcd for $\mathrm{C}_{28} \mathrm{H}_{49} \mathrm{~N}_{3} \mathrm{O}_{8}+\mathrm{H}$ : 276.1923). NMR and IR spectra were identical to those of its enantiomer 5.

\section{Acknowledgments}

The Swiss National Science Foundation (grants $n^{\circ} 20-63667.00$ and 2100-063567.00/1), the European COST (COST D13/0001/99) program, the "Office Fédéral de l'Education et de la Science" (Bern), the "Dirreción General de Investigación Científica y Técnica" of Spain (grant $\mathrm{n}^{\circ}$ BQU-2001-3779) and SOCRATES (EPFL/Sevilla) programs are gratefully acknowledged for financial support. We also thank Dr. Vladimir Kren of the Academy of Sciences of the Czech Republic for suggesting to us this investigation.

\section{References}

1. (a) Morenem, K. W.; Trimble, R. B.; Herscovics, A. Glycobiology 1994, 4, 113. (b) Varki, A. Glycobiology 1993, 3, 97. (c) Crocker, P. R.; Feizi, T. Curr. Opin. Struct. Biol. 1996, 6, 679. (d) Dwek, R. A. Chem. Rev. 1996, 96, 683.

2. (a) Kirby, A. J. Acc. Chem. Res. 1984, 17, 305. (b) Gorenstein, D. G. Chem. Rev. 1987, 87, 1047. (c) Sinnott, M. L. Chem. Rev. 1990, 90, 1171. (d) Jeong, J. H.; Murray, B. W.; Takayama, S.; Wong, C. H. J. Am. Chem. Soc. 1996, 118, 4227. (e) Ganem, B. Acc. Chem. Res. 1996, 29, 340. (f) Bols, M. Acc. Chem. Res. 1998, 31, 1. (g) Heightman, T. D.; Vasella, A. T. Angew. Chem., Int. Ed. 1999, 38, 750. (h) Ichikawa, Y.; Lin, Y. C.; Dumas, D. P.; Shen, G. J.; Garcia-Jonceda, E.; Williams, M. A.; Bayer, R.; Ketcham, C.; Walker, L. E.; Paulson, J. C.; Wong, C. H. J. Am. Chem. Soc. 1992, 114, 9283. (i) Qiao, L. Murray, B. W.; Shimazakin, M.; Schultz, J.; Wong, C. H. J. Am. Chem. Soc. 1996, 118, 7653. (j) Jefferies, I.; Bowen, B. R. Bioorg. Med. Chem. Lett. 1997, 7, 1171. (k) Palcic, M. M.; Heerze, L. D.; Srivastava, O. P.; Hindsgaul, O. J. Biol. Chem. 1989, 264, 17174.

3. (a) Fernandes, B.; Sagman, U.; Auger, M.; Demetrio, M.; Dennis, J. W. Cancer Res. 1991, 51, 718. (b) Robinson, K. M.; Begovic, M. E.; Rhinerhardt, M. E.; Heineke, E. W.; Ducep, J. B.; Kastner, P. R.; Marshall, F. N.; Danzin, C. Diabetes 1991, 40, 825. (c) Platt, F. M.; Reinkensmeier, G.; Dwek, R. A.; Butters, T. D. J. Biol. Chem. 1997, 272, 19365. (d) Lapierre, F.; Holme, K.; Lam, L.; Tressler, R. J.; Storm, N.; Wee, J.; Stack, R. J.; Castellot, J.; Tyrrell, D. J. Glycobiology 1996, 6, 355. (e) Mehta, A.; Zizmann, N.; Rudd, P. M.; Block, T. M.; Dwek, R. A. FEBS Lett. 1998, 430, 17. (f) Kolter, T. Angew. Chem., Int. Ed. 1997, 36, 1955. (g) Fan, Q. J.; Ishii, S.; Asano, N.; Suzuki, Y. Nat. Med. 1999, 5, 112. (h) Cox, T.; Lachman, R.; Hollack, C.; Aerts, J.; van Weely, S.; Hrebicek, M.; Platt, F.; Butters, T.; Dwek, R.; Moyses, C.; Gow, I.; Elstein, D.; Zimran, A. Lancet 2000, 355, 1481. 
4. (a) Stütz, A. E. Iminosugars as Glycosidase Inhibitors: Nojirimycin and Beyond; WileyVCH: Weinheim, 1999. (b) Asano, N.; Nash, R. J.; Molineux, R. J.; Fleet, G. W. J. Tetrahedron: Asymmetry 2000, 11, 1645. (c) Asano, N.; Nishida, M.; Kato, A.; Kizu, H.; Matsui, K.; Shimida, Y.; Itoh, T.; Baba, M.; Watson, A. A.; Nash, R. J.; de Q Lilley, X.; Watkin, D. J.; Fleet, G. W. J. J. Med. Chem. 1998, 41, 2565. (d) Martin, O. R.; Saavedra, O. M.; Xie, F.; Liu, L.; Picasso, S.; Vogel, P.; Kizu, H.; Asano, N. Bioorg. Med. Chem. Lett. 2001, 9, 1269.

5. (a) Jespersen, T. M.; Dong, W.; Sierks, M. R.; Skrydstrup, T.; Lundt, I.; Bols, M. Angew. Chem., Int. Ed. 1994, 33, 1778. (b) Ichikawa, Y.; Igarashi, Y. Tetrahedron Lett. 1995, 36, 4585. (c) Igarashi, Y.; Ichikawa, M.; Ichikawa, Y. Bioorg. Med. Chem. Lett. 1996, 6, 553. (d) Ichikawa, Y.; Igarashi, Y.; Ichikawa, M.; Suhura, Y. J. Am. Chem. Soc. 1998, 120, 5854. (e) Williams, S. J.; Hos, R.; Whiters, S. G. J. Am. Chem. Soc. 2000, 122, 2223. (f) Nishimura, Y.; Shitara, E.; Adachi, H.; Toyoshima, M.; Nakajima, M.; Okami, Y.; Takeuchi, T. J. Org. Chem. 2000, 65, 2. (j) Liu, H.; Liang, X.; Sфhoel, H.; Bülow, A.; Bols, M. J. Am. Chem. Soc. 2001, 123, 5116, (h) Jensen, H.; Bols, M. J. Chem. Soc., Perkin Trans. 1 2001, 905.

6. (a) Fleet, G. W. J.; Nicholas, S. J.; Smith, P. W.; Evans, S. V.; Fellows, L. E.; Nash. R. J. Tetrahedron Lett. 1985, 26, 3127. (b) Asano, N.; Oseki, K.; Kizu, H.; Matsui, K. J. Med. Chem. 1994, 37, 3701. (c) Asano, N.; Nishida, M.; Miyauchi, M.; Ikeda, K.; Yamamoto, M.; Kizu, H.; Kameda, Y.; Watson, A. A.; Nash, R. J.; Fleet, G. W. J. Phytochemistry 2000, 53, 379.

7. (a) Saotome, C.; Kanie, Y.; Kanie, O.; Wong, C. H. Bioorg. Med. Chem. 2000, 8, 2249 references cited therein. (b) Saotome, C.; Wong, C. H.; Kanie, O. Chem. Biol. 2001, 8, 1061.

8. (a) Popowycz, F.; Gerber-Lemaire, S.; Demange, R.; Rodriguez-García, E.; CarmonaAsenjo, A. T.; Robina, I.; Vogel, P. Bioorg. Med. Chem. Lett. 2001, 11, 2489. (b) Popowycz, F.; Gerber-Lemaire, S.; Rodriguez-García, E.; Schütz, C.; Vogel, P. Helv. Chim. Acta, in press.

9. (a) White, S. L.; Nagai, T.; Akiyama, S. K.; Reeves, E. J.; Grzegorzewski, K.; Olden, K. Cancer Commun. 1991, 3, 83. (b) Olden, K.; Breton, P.; Grzegorzewski, K.; Yasuda, Y.; Gause, B. L.; Oredipe, O. A.; Newton, S. A.; White, S. L. Pharmacol.Ther. 1991, 50, 285. (c) Asano, N. J. Enzyme Inhibition 2000 15, 215. (d) Carver, J.; Dennis, J. W.; Shah, P. US Patent 5773239A, 30 Jun. 1998; Chem. Abstr. 1998, 129, 95683.

10. Colegate, S. M.; Dorling, P. R.; Huxtable, C. R. Austr. J. Chem. 1979, 32, 2257.

11. Elbein, A. D.; Molyneux, R. D. Iminosugars as Glycosidase Inhibitors; Nojirimycin and Beyond, A. E. Stütz, Ed; Wiley-VCH: Weinheim, 1999, Chapt.11, pp 216-251.

12. (a) Goss, P. E.; Baptiste, J.; Fernades, B.; Baker, M.; Dennis, J. W. Cancer Res. 1994, 54, 1450. (b) Goss, P. E.; Reid, C. L.; Bailey, D.; Dennis, J. W. Clin. Cancer Res. 1997, 3, 1077.

(c) P. D. Rye, N. V. Bovin, E. V. Vlasova, R. A. Walker, Glycobiology 1995, 5, 385.

13. (a) Pearson, W. H.; Guo, L. Tetrahedron Lett. 2001, 42, 8267. (b) Pearson, W. H.; Perlmutter, D. H. Tetrahedron Lett. 2001, 42, 8273. 
14. (a) Aoyagi, T.; Yamamoto, T.; Kojiri, K.; Morishima, H.; Nagai, M.; Hamada, M.; Takeuchi, T.; Umezawa, H. J. Antibiot. 1989, 42, 883. (b) Morishima, H.; Kojiri, K.; Yamamoto, T.; Aoyagi, T.; Nakamura, H.; Iitaka, Y. J. Antibiot. 1989, 42, 100. (c) Tropea, J. E.; Kaushal, G. P.; Pastuszak, I.; Mitchell, M.; Aoyagi, T.; Molyneux, R. J.; Elbein, A. D. Biochem. 1990, 29, 10062. (d) Berecibar, A.; Grandjean, C.; Siriwardena, A. Chem. Rev. 1999, 99, 779.

15. Ogawa, S.; Morikawa, T. Bioorg. Med. Chem. Lett. 2000, 10, 1047.

16. (a) Ogawa, S.; Morikawa, T. Eur. J. Org. Chem. 2000, 1759. (b) Ogawa, S.; Morikawa, T. Bioorg. Med. Chem. Lett. 1999, 9, 1499. (c) Wong, C. H.; Provencher, L.; Porco, J. A.; Jung, S. H.; Wang, Y. F.; Chen, L. R.; Wang, R.; Steensma, D. H. J. Org. Chem. 1995, 60, 1492.

17. Winkler, D. A. J. Med. Chem. 1996, 39, 4332.

18. See e.g. Davis, B. G.; Brandstetter, T. W.; Hackett, L.; Winchester, B. G.; Nash, R. J.; Watson, A. A.; Griffiths, R. C.; Smith, C.; Fleet, G. W .J. Tetrahedron 1999, 55, 4489.

19. (a) Andersen, S. M.; Ekhart, C.; Lundt, I.; Stütz, A. E. Carbohydr. Res. 2000, 326, 22. (b) Kim, Y. J.; Takatsuki, A.; Kogoshi, N.; Kitahara, T. Tetrahedron 1999, 55, 8353. (c) Joubert, M.; Defoin, A.; Tarnus, C.; Streith, J. Synlett 2000, 1366.

20. See e.g. (a) Lipper, R. A. Modern Drug Discovery 1999, 55. (b) Lipinsky, C. A.; Lombardo, F.; Dominy, B. W.; Feeney, P. J. Adv. Drug Delivery Rev. 1997, $23,3$.

21. Howard, S.; He, S.; Whithers, S. G. J. Biol. Chem. 1998, 273, 2067.

22. Abdel-Magid, A. F.; Carson, K. G.; Harris, B. D.; Maryanoff, C. A.; Shah, R. D. J. Org. Chem. 1996, 61, 3849.

23. (a) Thompson, D. K.; Hubert, C. N.; Wightman, R. H. Tetrahedron 1993, 49, 3827. (b) Cardona, F.; Robina, I.; Vogel, P. J. Carbohydr. Chem. 2000, 19, 555.

24. Robina, I.; Gearing, R. P.; Buchanan, J. G.; Wightman, R. H. J. Chem. Soc., Perkin Trans. 1 1990, 2622;

25. (a) Brandi, A.; Cicchi, S.; Cordero, F. M.; Frigneoli, B.; Goti, A.; Picasso, S.; Vogel, P. J. Org. Chem. 1995, 60, 6806. (b) Picasso, S.; Chen. Y.; Vogel, P. Carbohydr. Lett. 1994, 1, 1.

26. Saul, R.; Chambers, J. P.; Molyneux, R. J.; Elbein, A. D. Arch. Biochem. Biophys. 1983, 221, 593. 